\title{
Energy spectrum of interplanetary magnetic flux ropes and its connection with solar activity
}

\author{
D. J. Wu ${ }^{1}$, H. Q. Feng ${ }^{2}$, and J. K. Chao ${ }^{3}$ \\ 1 Purple Mountain Observatory, 2 West Beijing Road, Nanjing 210008, PR China \\ e-mail: djwu@pmo.ac.cn \\ 2 Department of Physics, Luoyang Normal College, Luoyang, PR China \\ 3 Institute of Space Science, National Central University, Chungli 32001, Taiwan
}

Received 30 November 2007 / Accepted 17 January 2008

\begin{abstract}
Context. Recent observations of the solar wind show that interplanetary magnetic flux ropes (IMFRs) have a continuous scaledistribution from small-scale flux ropes to large-scale magnetic clouds.

Aims. In this work, we investigate the energy spectrum of IMFRs and its possible connection with solar activity.

Methods. In consideration of the detectable probability of an IMFR to be proportional to its diameter, the actual energy spectrum of IMFRs can be obtained from the observed spectrum based on spacecraft observations in the solar wind.

Results. It is found that IMFRs have a negative power-law spectrum with an index $\alpha=1.36 \pm 0.03$, which is similar to that of solar flares, and is probably representative of interplanetary energy spectrum of coronal mass ejections (CMEs), that is, the energy spectrum of interplanetary CMEs (ICMEs). This indicates that the energy distribution of CMEs has a similar negative power-law spectrum. In particular, there are numerous small-scale CMEs in the solar corona, and their interplanetary consequences may be directly detected in situ by spacecraft in the solar wind as small-scale IMFRs, although they are too weak to appear clearly in current coronagraph observations.

Conclusions. The presence of small-scale CMEs, especially the energy spectrum of CMEs is potentially important for understanding both the solar magneto-atmosphere and CMEs.
\end{abstract}

Key words. Sun: activity - Sun: coronal mass ejections (CMEs) - Sun: flares - Sun: solar wind

\section{Introduction}

Coronal mass ejections (CMEs) are believed to be created by a force imbalance that triggers the eruption of large closed magnetic structures in the solar corona, carrying away the local magnetic field and embedded plasma. These structures move into the solar wind and can be detected as interplanetary CMEs (ICMEs) by spacecrafts (e.g., Kahler 1992; Cane \& Richardson 2003; Schwenn et al. 2005). Magnetic clouds (MCs) are an important subset of ICMEs and are characterized by depressed proton temperatures and enhanced magnetic fields that rotate slowly through a large angle during a day (Burlaga et al. 1981; Burlaga 1991). The magnetic configuration of MCs is amenable to simple force-free field modeling and has a flux-rope geometry (Goldstein 1983). Although it is difficult to determine the global topology of a MC, its local magnetic structure may be fitted simply by the so-called Lundquist solution (Burlaga 1988; Lepping et al. 1990). The result shows that MCs are large-scale interplanetary magnetic flux ropes (IMFRs) with typical diameters of $0.2 \sim 0.3 \mathrm{AU}$.

Nearly twenty years after MCs were discovered, Moldwin et al. (2000) identified several small-scale IMFRs with a duration $\sim 1 \mathrm{~h}$ and a diameter $\sim 0.01 \mathrm{AU}$ in the solar wind observations of the IMP 8 and WIND spacecrafts. These small-scale IMFRs have similar magnetic structures to those of MCs, but different plasma behaviors. Without a systematic survey of solar wind data, Moldwin et al. did not find any intermediate-sized
IMFRs with durations of several hours. This resulted in a bimodal size-distribution of IMFRs consisting of two populations, large-scale MCs and small-scale IMFRs, and it is suggested that the small-scale IMFRs result from magnetic reconnection in the solar wind as opposed to in the solar corona (Moldwin et al. 2000). As pointed out by the authors, however, it is still doubtful whether IMFRs have an interplanetary or a coronal origin. In a recent work, Feng et al. (2007) systematically surveyed the solar wind data of the WIND spacecraft between 1995-2001, and identified 144 IMFRs. Feng et al. found a duration-distribution continuously ranging from tens of minutes to tens of hours, and a size-distribution from 0.004 to $0.6 \mathrm{AU}$. Half of them have durations of less than 10 hours and diameters of less than $0.1 \mathrm{AU}$.

In this letter, we analyze the energy distribution of IMFRs and their connection with solar activity. The result shows that they have a continuous energy distribution, ranging from $\sim 10^{19}$ to $\sim 10^{24} \mathrm{~J}$, and that the energy distribution can be fitted well by a negative power-law spectrum with an index $\sim 1.36 \pm 0.03$, which is similar to, but slightly lower than, the index of solar flares. We suggest that this energy spectrum probably is representative of that of ICMEs, that is, the interplanetary energy spectrum of CMEs. The result implies that CMEs also have a negative powerlaw energy spectrum similar to that of flares. In particular, there are more small-scale CMEs in the solar corona, although they are too weak to appear clearly in the current coronagraph observations as ordinary CMEs. The energy spectrum of CMEs and 
the presence of small-scale CMEs are potentially important for understanding the solar magneto-atmosphere and CMEs.

\section{Energy spectrum of IMFRs}

The total energy of an IMFR consists of the thermal energy of the plasma embedded in the IMFR, the kinetic energy of its movement through the interplanetary medium, and its magnetic energy. Protons, electrons, and helium ions (i.e. $\alpha$ particles) are major plasma species in the IMFR. Taking the abundance ratio of helium ions to protons as $5 \%$, that is, $n_{\mathrm{He}}=0.05 n_{\mathrm{p}}$, where $n_{\mathrm{He}(\mathrm{p})}$ is the density of helium ions (protons), one has the electron density $n_{\mathrm{e}}=n_{\mathrm{p}}+2 n_{\mathrm{He}}=1.1 n_{\mathrm{p}}$ because of the charge neutrality condition. These species often have different temperatures. For example, Liu et al. $(2005,2006 a)$ find that in ICME cases electron and helium ion temperatures are about three and four times of proton temperature, respectively. In result, the thermal energy density in the IMFR can be estimated by

$\mathcal{E}_{\mathrm{T}}=\left(n_{\mathrm{p}}+3 n_{\mathrm{e}}+4 n_{\mathrm{He}}\right) 3 k_{\mathrm{B}} T_{\mathrm{p}} / 2$,

where $n_{\mathrm{p}}$ and $T_{\mathrm{p}}$ are the mean proton density and temperature for the IMFR, respectively. In consideration of the IMFR moving through the ambient solar wind in the interplanetary medium, the kinetic energy density can be estimated as (Feng et al. 2007)

$\mathcal{E}_{\mathrm{K}}=\left(n_{\mathrm{p}}+4 n_{\mathrm{He}}\right) m_{\mathrm{p}}\left(V_{\mathrm{FR}}-V_{\mathrm{SW}}\right)^{2} / 2$,

where $V_{\mathrm{FR}}$ and $V_{\mathrm{SW}}$ are the mean IMFR speed and the ambient solar wind speed, respectively. Some evidence from observations and simulations suggests that IMFRs may be flattened and distorted due to their interaction with the ambient solar wind (e.g. Liu et al. 2006b). This probably causes their magnetic configuration to deviate from cylindrically symmetric ropes. For the sake of simplicity, we assume that the distortion does not significantly affect the estimate of the magnetic energy content. According to the constant $\alpha$ force-free field model, which fits well the magnetic configuration of IMFRs (Feng et al. 2007), the mean magnetic energy density of the IMFR can be calculated as follows:

$\mathcal{E}_{\mathrm{B}}=\int_{0}^{R_{0}}\left(B^{2} / 2 \mu_{0}\right) 2 \pi R \mathrm{~d} R /\left(\pi R_{0}^{2}\right)$,

where $R_{0}$ is the IMFR radius and $B_{0}$ is the axial magnetic field intensity of the IMFR.

It is difficult to determine the global configuration of a MC, although its local magnetic structure can be fitted simply by the cylindrically-symmetric, constant- $\alpha$, force-free field with the Lundquist solution (Burlaga 1988; Lepping et al. 1990). Some characteristic energetic particle signature for ICMEs (e.g. a flare particle onset within ICMEs), however, is consistent with the presence within the ICMEs of regions of looped magnetic field lines rooted at the Sun at both ends (Kahler \& Reames 1993; Cane \& Richardson 2003). In this case, a simple assumption about the global topology of IMFRs is that the flux rope ends at a pair of compact regions on the surface of the Sun and has an axial length of $2<L_{0}<\pi$ AU when arriving at the Earth. And the total energy of an IMFR can be estimated by

$E=\left(\mathcal{E}_{\mathrm{T}}+\mathcal{E}_{\mathrm{K}}+\mathcal{E}_{\mathrm{B}}\right) \pi R_{0}^{2} L_{0}$.

Based on the survey of Feng et al. (2007), Fig. 1 shows the number of the IMFRs that have an energy larger than $E, N(E)$, where an effective length of $L_{0}=2.5 \mathrm{AU}$ has been used in the energy calculation of Eq. (4). The observed IMFR energies range

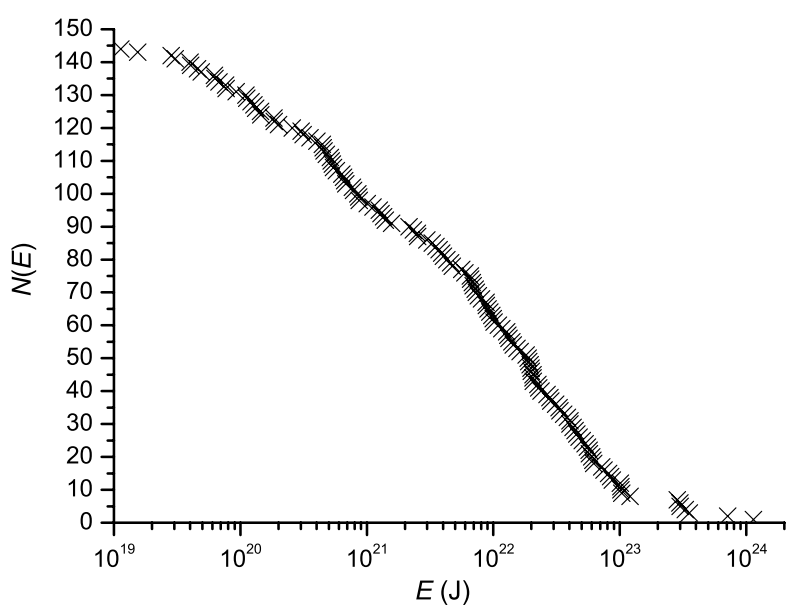

Fig. 1. The cumulate distribution of IMFRs: the recurrence rate of IMFRs with energy larger than $E$.

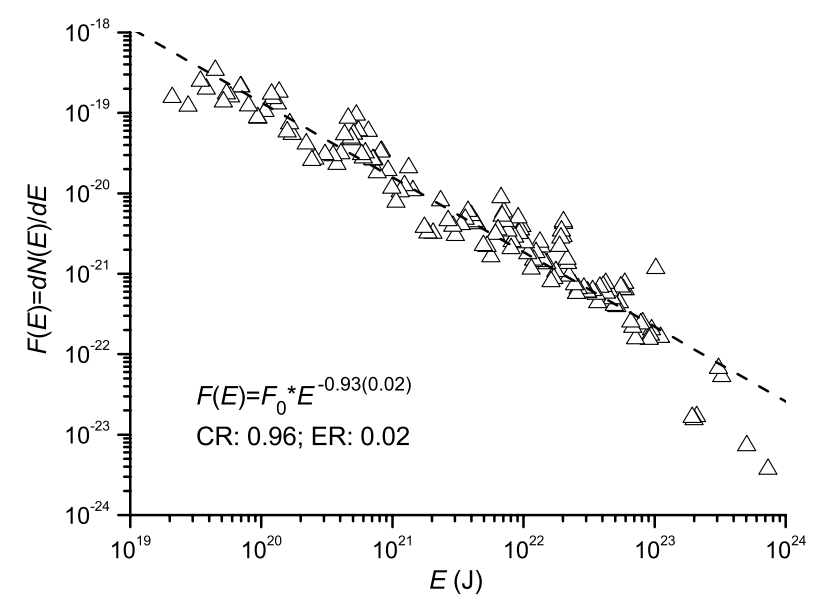

Fig. 2. The differential distribution of IMFRs: the recurrence rate in the unit energy of IMFRs with energy $E$.

from $\sim 10^{19}$ through $\sim 10^{24} \mathrm{~J}$, and the energy distribution, that is, the recurrence rate in the unit energy can be calculated from the derivation of $N(E)$ with respect to $E$. In order to avoid the singularity due to the possibility of any two neighboring IMFRs with almost the same energy, we take the three-point average of $N(E)$ to calculate the derivation $\delta N(E) / \delta E$. The result is plotted in Fig. 2. From Fig. 2, the energy distribution of the IMFRs, $F(E)=\mathrm{d} N(E) / \mathrm{d} E$, can be fitted by a negative power-law spectrum with an index of $0.94 \pm 0.02$, that is, the observed energy spectrum

$F(E)=F_{0} E^{-0.93 \pm 0.02}$.

The fitting has a high correlation factor $(\sim 0.96)$ and a low relative error $(\sim 2 \%)$ as shown in Fig. 2.

In consideration of the geometric effect of flux rope sizes on the detectable probability, however, an IMFR with a larger diameter is more easily observed by spacecraft located in the solar wind because it has a larger "collision cross-section" with the spacecraft. In consequence, the observable probability of an IMFR is in direct proportion to the rope diameter, $D$. This indicates that the actual energy spectrum $\mathcal{F}(E)$ of IMFRs should be related to the observed spectrum $F(E)$ by

$F(E) \propto \mathcal{F}(E) D$. 


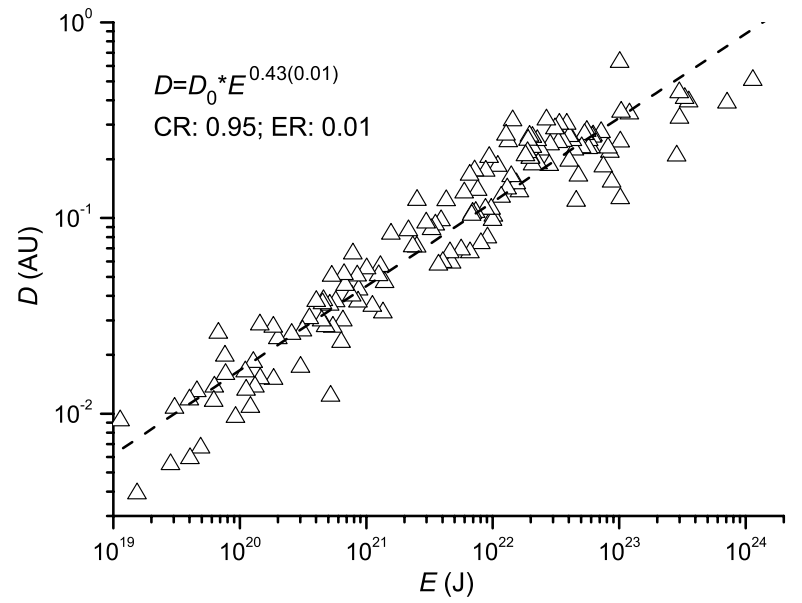

Fig. 3. The diameter-energy relation for IMFRs.

Figure 3 plots the diameters of the IMFRs versus their energies. From Fig. 3, the diameter-energy relation of the IMFRs, $D(E)$, can be fit well by

$D \propto E^{0.43 \pm 0.01}$

with a high correlation factor of 0.95 and low relative error of $2.5 \%$. Combining Eqs. (5)-(7), we obtain the actual energy spectrum of IMFRs as follows:

$\mathcal{F}(E)=\mathcal{F}_{0} E^{-1.36 \pm 0.03}$.

\section{Possible connection between IMFR spectrum and solar activity: small-scale CMEs}

Solar eruptions frequently produce both CMEs and flares, called flare-associated CMEs, and the ubiquitous magnetic reconnection in solar magneto-atmospheres is believed to be a common triggering source for CMEs and flares (e.g., Kahler 1992; Andrews \& Howard 2001; Moon et al. 2002). Observations of flares show that their distribution in energy has a negative powerlaw spectrum in a wide range from nano- and microflares to large flares, namely $\mathcal{N}(E)=\mathcal{N}_{0} E^{-\alpha}$, with a spectrum index $1.5<\alpha<2$ (Hudson 1991; Craig 2001). We think that a negative power-law energy spectrum is probably a common characteristic of solar eruptions. In particular, CMEs have a negative power-law energy spectrum similar to flares. This indicates that there are more small-scale CMEs in the solar corona, although they can not be observed directly by the current observational technique because they are too weak to appear clearly in the coronagraph observations. In particular, the small-scale IMFRs are probably the interplanetary manifestation of smallscale CMEs, and the energy spectra of IMFRs are representative of those of ICMEs in the solar wind. The energy spectra of ICMEs have dynamically direct connectivity to the energy spectra of CMEs in the solar corona (Chen \& Garren 1993; Howard et al. 2007). In this connection, the identified small-scale IMFRs can be treated as possible evidence for the existence of smallscale CMEs.

In fact, there is evidence for small solar eruptions, such as X-ray bright sigmoidal structures (Sterling \& Hudson 1997), X-ray plasmoids (Shibata et al. 1995; Ohyama \& Shibata 1998; Einaudi 2001), and tiny jets observed recently by the Solar-B
(Shibata et al. 2007), although there are no direct observations of small-scale CMEs. These small eruptions can be triggered by ubiquitous magnetic reconnection in solar magnetoatmospheres. Small-scale CMEs may be direct by-products of the ubiquitous magnetic reconnection. In particular, in a recent work, Mandrini et al. (2005a,b) have provided some direct evidence for a small eruption observed on the solar disk center, linked to a small-scale IMFR, with a duration $\sim 4 \mathrm{~h}$ and a diameter $\sim 0.03 \mathrm{AU}$. This evidence includes the timing, the same orientation of the coronal loop and the rope relative to the ecliptic, the same magnetic field direction and magnetic helicity sign in the coronal loop and in the rope, and comparable magnetic flux measured in the dimming regions and in the rope. Finally, the pre- to post-event change of magnetic helicity in the solar corona is found to be comparable to the helicity content of the rope.

It is worth noticing that the spectrum index for ICMEs is slightly lower than for flares. One possible reason for this lowering is the presence in the solar wind of Alfvénic fluctuations (Mariani \& Neubauer 1990) and other small-scale structures associated with large variations in the magnetic field direction (Marsch 1991). The magnetic configuration of small-scale ICMEs more easily are disturbed or distorted by these interplanetary magnetic fluctuations so that it is more difficult to identify them as simple flux ropes by the least squares fitting technique. This results in fewer observed small-scale IMFRs relative to the actual small-scale CMEs from the solar corona. As a consequence, the energy distribution of IMFRs has a spectral index lower than that of CMEs.

The dynamical evolution of CMEs propagating through the interplanetary medium can probably influence their energy distribution. On the one hand, the resistance and deceleration encountered by an ICME in its interplanetary propagation depend on its initial velocity and scale-size (Cargill 2004; Tappin 2006; Howard et al. 2007). This results in its kinetic energy to depend on its initial conditions in the solar corona. On the other hand, the electrodynamic coupling between an ICME and solar wind magnetic fields can also considerably influence its motion and magnetic configuration, and hence its kinetic and magnetic energies (Chen 1989; Chen \& Garren 1993; Howard et al. 2007). It is rather difficult, however, to evaluate the energy variation of ICMEs during their interplanetary propagating.

Furthermore, it is also possible that CMEs originally have a different spectrum index from flares so that their interplanetary consequences, ICMEs, have a different index. In any case, CMEs and flares represent different physical phenomena, although they are frequently associated with magnetic reconnection. In fact, flares are the rapid enhancement of energetic particles and electromagnetic radiation but CMEs are ejections of magnetized plasmas. Flares and CMEs probably have different recurrence rates in large and small scales so that they have different energy spectrum indices.

Finally, it is necessary to point out that the present work cannot absolutely exclude the possibility of the interplanetary origin of small-scale IMFRs, although they and MCs that originate from CMEs have a common energy spectrum. Maybe, it is truer that a part of them originates from small-scale CMEs and the other part has interplanetary origin. However, it is rather difficult and needs further work to distinguish clearly the two origins.

\section{Discussion and summary}

As noticed by Moldwin et al. (2000), besides the dramatic disparity in size, the small-scale IMFRs have two important 
differences from MCs although their magnetic structures are very similar to MCs. One is the different proton temperature behaviors. The proton temperature in MCs is consistently lower than the ambient solar wind but the temperature changes very little across the small-scale IMFRs. The other one is the lack of expansion of the small-scale IMFRs, but MCs usually are still expanding at $1 \mathrm{AU}$. One of possible explanations is that the small-scale IMFRs usually have weaker magnetic fields than MCs. On the one hand, plasma particles more easily diffuse and mix with each other between the small-scale IMFRs and the ambient solar wind. On the other hand, the weaker magnetic pressures inside the small-scale IMFRs are more difficult to drive expansion. Although interplanetary magnetic reconnection occurring near heliospheric current sheets (HCSs) or MCs (Moldwin et al. 1995, 2000; Wei et al. 2003) is still one possible origin for small-scale IMFRs, there is no evident correlation between the small-scale IMFRs and HCSs or MCs (Feng et al. 2007).

In summary, in this letter we present the energy spectrum of IMFRs based on the observations of IMFRs, a negative powerlaw spectrum with an index of $1.36 \pm 0.03$, which is similar to that of flares but a slightly lower index. We also discuss the possible connection between the IMFR energy spectrum and solar eruptions, and propose that a negative power-law energy spectrum is probably a common characteristic of solar eruptions including flares and CMEs. This indicates that there are more smallscale CMEs produced in the solar corona and that they can be detected by spacecraft in the solar wind as their interplanetary manifestation, small-scale IMFRs, although they are too weak to appear clearly in current coronagraph observations. The presence of small-scale CMEs, especially the energy spectrum of CMEs is potentially important not only for improving current CME models, which are developed for large-scale magnetic configurations in the corona, but also for understanding the solar magneto-atmospheric dynamics.

Acknowledgements. This project is supported by the NSFC programs 10425312 and 40574065, the NKBRSF program 2006CB806302, and the CAS project KJCX2-YW-T04. The authors also are grateful to the referee for his/her constructive comments.

\section{References}

Andrews, M. D., \& Howard, R. A. 2001, Space. Sci. Rev., 95, 147 Burlaga, L. F. 1988, J. Geophys. Res., 93, 7217

Burlaga, L. F. 1991, in Physics of the Inner Heliosphere II: Particles, Waves, and Turbulene, ed. R. Schwenn, \& E. Marsch (New York: Springer), 1

Burlaga, L. F., Sittler, E., Mariani, F., \& Schwenn, R. 1981, J. Geophys. Res., 86,6673

Cane, H. V., \& Richardson, I. G. 2003, J. Geophys. Res., 108, 1156

Cargill, P. J. 2004, Sol. Phys., 221, 135

Chen, J. 1989, ApJ, 338, 453

Chen, J., \& Garren, D. A. 1993, Geophys. Res. Lett., 20, 2319

Craig, I. J. D. 2001, Sol. Phys., 202, 109

Einaudi, G., Chibbaro, S., Dahlburg, R. B., \& Velli, M. 2001, ApJ, 547, 1167

Feng, H. Q., Wu, D. J., \& Chao, J. K. 2007, J. Geophys. Res., 112, A02102

Goldstein, H. 1983, in Solar Wind Five, ed. M. Neugebauer (NASA Conference Publ. 2280), 731

Howard, T. A., Fry, C. D., Johnston, J. C., \& Webb, D. F. 2007, ApJ, 667, 610

Hudson, H. S. 1991, Sol. Phys., 133, 357

Kahler, S. W. 1992, ARA\&A, 30, 113

Kahler, S. W., \& Reames, D. V. 1991, J. Geophys. Res., 96, 9419

Lepping, R. P., Burlaga, L. F., \& Jones, J. A. 1990, J. Geophys. Res., 95, 11957

Liu, Y., Richardson, J. D., \& Belcher, J. W. 2005, Planet. Space Sci., 53, 3

Liu, Y., Richardson, J. D., Belcher, J. W., et al. 2006a, J. Geophys. Res., 111, A01102

Liu, Y., Richardson, J. D., Belcher, J. W., et al. 2006b, J. Geophys. Res., 111, A12S03

Mandrini, C. H., Pohjolainen, S., \& Dasso, S. 2005a, A\&A, 434, 725

Mandrini, C. H., Pohjolainen, S., Dasso, S., et al. 2005b, Adv. Space Sci., 36, 1579

Mariani, F., \& Neubauer, F. M. 1990, in Physics of the Inner Heliosphere I: Large-Scale Phenomena, ed. R. Schwenn, \& E. Marsch (New York: Springer), 183

Marsch, E. 1991, in Physics of the Inner Heliosphere II: Particles, Waves, and Turbulene, ed. R. Schwenn, \& E. Marsch (New York: Springer), 159

Moldwin, M. B., Phillips, J., Gosling, J., et al. 1995, J. Geophys. Res., 100, 19903

Moldwin, M. B., Ford, S., Lepping, R., Slavin, J., \& Szabo, A. 2000, Geophys. Res. Lett., 27, 57

Moon, Y. J., Choe, G. S., \& Wang, Haimin 2002, ApJ, 581, 694

Ohyama, M., \& Shibata, K. 1998, ApJ, 499, 934

Schwenn, R., Dal Lago, A., Huttunen, E., \& Gonzalez, W. D. 2005, Ann. Goephysicae, 23, 1033

Shibata, K., Masuda, S., \& Shimojo, M. 1995, ApJ, 451, L83

Shibata, K., et al. 2007, presented in The 4th AOGS Annual Conference: ST02 Session, Bangkok, Thailand

Sterling, A. C., \& Hudson, H. S. 1997, ApJ, 491, L55

Tappin, S. J. 2006, Sol. Phys., 233, 233

Wei, F. S., Hu, Q., Feng, X. S., \& Fan, Q. L. 2003, Space Sci. Rev., 107, 107 